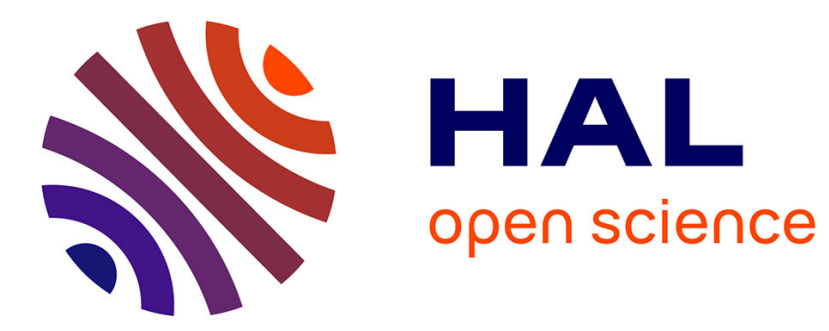

\title{
Un professeur de lettres chrétien au pays de Moab
} Julien Aliquot

\section{To cite this version:}

Julien Aliquot. Un professeur de lettres chrétien au pays de Moab. Topoi Orient - Occident, 2018, 22 (1), pp.331-340. halshs-02413354

\section{HAL Id: halshs-02413354 https://shs.hal.science/halshs-02413354}

Submitted on 31 Jan 2020

HAL is a multi-disciplinary open access archive for the deposit and dissemination of scientific research documents, whether they are published or not. The documents may come from teaching and research institutions in France or abroad, or from public or private research centers.
L'archive ouverte pluridisciplinaire HAL, est destinée au dépôt et à la diffusion de documents scientifiques de niveau recherche, publiés ou non, émanant des établissements d'enseignement et de recherche français ou étrangers, des laboratoires publics ou privés. 


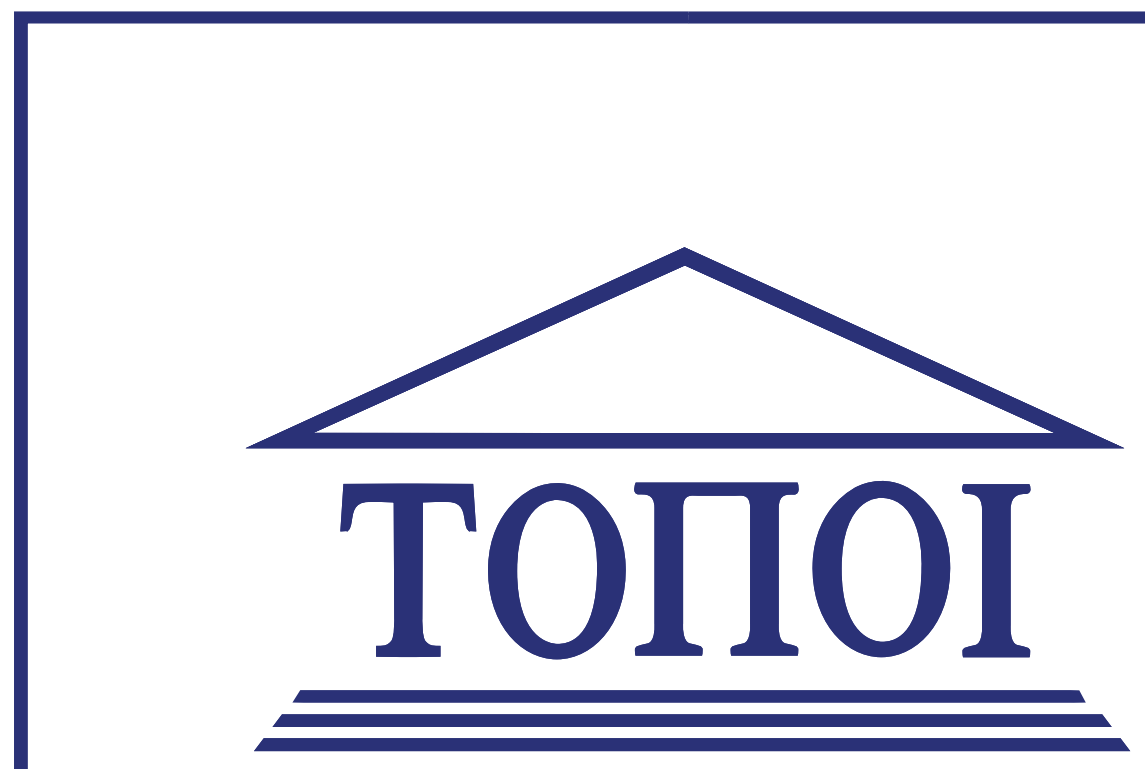

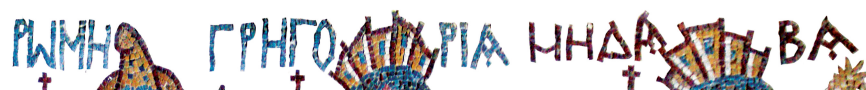

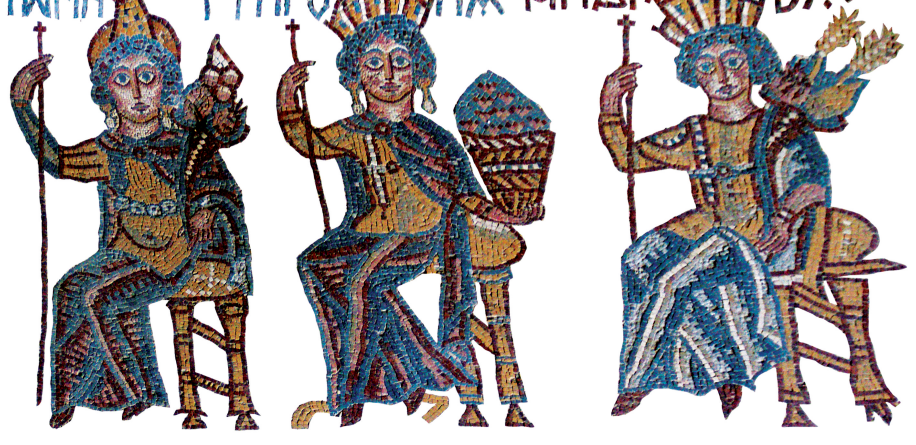

Volume 22/1 



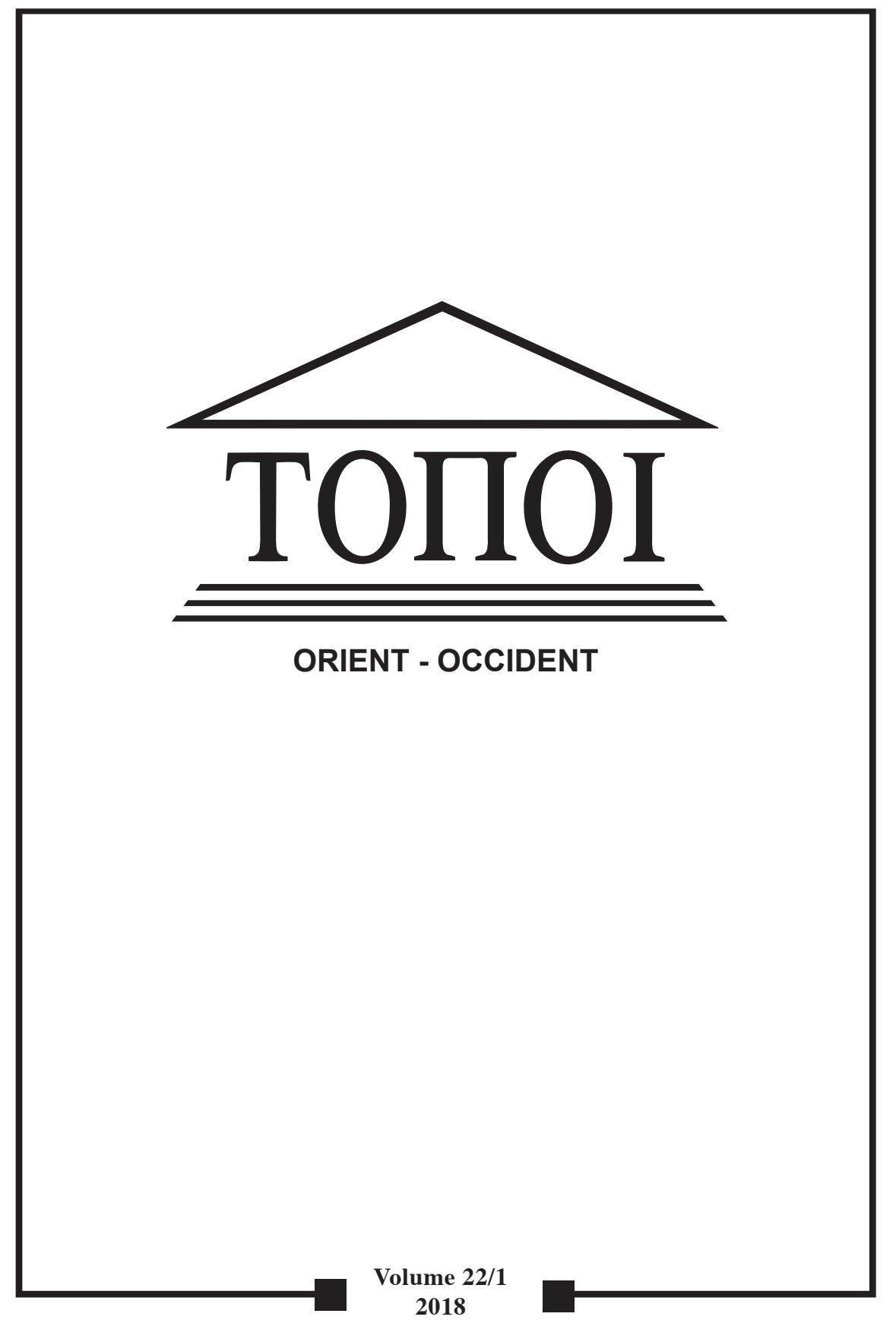



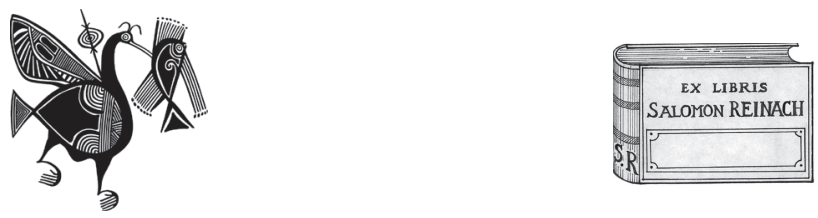

Ouvrage publié avec le concours

de la Société des Amis de la Bibliothèque Salomon Reinach

Comité d'honneur (au 01.01.2019):

Jean Andreau, Alexandre Farnoux, Ian Morris, †Georges Rougemont, Catherine VIRLOUVET

Comité de Rédaction (au 01.01.2019):

Marie-Françoise Boussac, Roland Étienne, Pierre-Louis Gatier, Jean-François SALLES, Laurianne MartinEZ-SÈve, Jean-Baptiste Yon

Responsable de la Rédaction: Marie-Françoise BoussaC

Adjoint : Jean-Baptiste YON

Maison de l'Orient et de la Méditerranée - Jean Pouilloux

5/7 rue Raulin, F-69365 Lyon Cedex 07, France

marie-francoise.boussac@mom.fr

www.topoi.mom.fr

https://www.persee.fr/collection/topoi

Diffusion : De Boccard Édition-Diffusion, 4 rue de Lanneau, F-75005 Paris

Topoi. Orient-Occident 22, Lyon (2018)

ISSN : $1161-9473$

Illustration de couverture: Mosaïque inscrite de Madaba I. Jordanie 2, 128 (C) Julien Aliquot CNRS/HiSoMA 2010.

Illustration du dos : Détail. 


\section{SOMMAIRE}

\section{Fascicule 1}

$\begin{array}{lr}\text { Sommaire } & 5-7\end{array}$

$\begin{array}{lr}\text { Index des auteurs } & 9-10\end{array}$

Dossier «Perles et écaille» (éd. P. Schneider)

P. SCHNEIDER, «Introduction»

J.TRINQUIER, «De la tortue marine à l'écaille: un matériau 'indien' du luxe romain»

P.Descotes, «Amour du luxe et amour de Dieu: la perle chez les auteurs latins chrétiens»

N. COQuery, «L'objet de luxe dans l'Europe moderne: définitions, appropriation, diffusion»

\section{Dossier «Épigraphie et histoire de la Jordanie antique et médiévale»} (éds J.Aliquot, P.-L.Gatier, J.-B. Yon)

J. Aliquot, P.-L.Gatier, J.-B.Yon, «Introduction»

J. NoRRIS, «Dushara dans une inscription thamoudique B de la région du Wādī Ramm (Jordanie du Sud)»

P.-L.GATIER, «Antioche du Chrysorhoas»

J. SEIGNE, «À propos de quelques inscriptions de Gérasa»

J. SeIGNE, «La dédicace de l'arc d'Hadrien à Gérasa»

N.BADER, J.-B.YON, «Une épigramme de la région de Gadara»

G.AGostI, «Le iscrizioni metriche e il ruolo della paideia classica in Giordania»

J. Aliquot, «Un professeur de lettres chrétien au pays de Moab»

N.BADER, «New Greek inscriptions from Wadi al-Rayyan in North-West Jordan»

\section{Études de géographie historique}

P.-O.LEROY, «Alexandre et les cataractes du Tigre. Étude terminologique

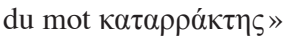

P. Arnaud, «Mallos, Antioche du Pyrame, Magarsus et le Stadiasme de la Grande Mer: toponymie historique, aléas politiques et compilation d'un périple» 


\section{SOMMAIRE}

\section{Fascicule 2}

Sommaire

$451-452$

\section{Chroniques bibliographiques, comptes rendus longs}

X.Lafon, A. Marzano et G.P.R. Métraux (dir.), The Roman Villa in the Mediterranean Basin. Late Republic to Late Antiquity (2018)

453-468

J.CAPELLE et al., H.P. Isler, Antike Theaterbauten: ein Handbuch (2017)

469-499

\section{Comptes rendus}

\section{Méditerranée gréco-romaine}

M. Gras, X. Charalambidou, C. Morgan, Interpreting the 7th Century BC (2017) 501-508

C. Constantakopoulou, A. Mazarakis Ainian (éd.), Les sanctuaires archä̈ques des Cyclades (2018)

S. Maudet, G. Olcese, «Pithecusan workshops » (2017)

A. JACQUEMIN, M.B. Hatzopoulos, La mort de Philippe II. Une étude des sources (2018)

M. SARTRE, K.Erickson, The early Seleukids, their Gods and their coins (2019); K.Erickson (éd.), The Seleukid Empire. War within the Family (2018)

P. SÁnchez, F.Canali De Rossi, Le relazioni diplomatiche di Roma, vol. VIII. La crisi dinastica macedone (2018)

Ch. Doyen, M. Rizzi, Marktbezogene Gesetzgebung im späthellenistischen Athen (2017)

$\mathrm{Cl}$. HasenoHR, P.Ernst, Recherches sur les pratiques culturelles des Italiens à Délos aux II et ${ }^{e r}$ siècles a.C. (2019)

A. Vlamos, C. Constantakopoulou, Aegean interactions. Delos and its Networks in the Third Century (2017)

N. Hudson, C. Harlaut, J. Hayes, Pottery in Hellenistic Alexandria (2018)

S. Anderson-Stojanovic, S.A. James, Corinth VII.7. Hellenistic Pottery (2018)

$569-575$

G. Finkielsztejn, G. Cancardeş-Şenol, Lexicon of Eponym Dies on Rhodian Amphora Stamps, Vol. 1-4 (2015-2017)

A. Marangou, A.K. Şenol, Commercial Amphorae in the Graeco-Roman Museum of Alexandria (2018)

Cr. Viglietti, D. Hoyer, Money, Culture, and Well-Being in Rome's Economic Development (2018)

Cl. BADY, D. Carrangeot, B. Laurioux et V. Puech (dir.), Rituels et cérémonies de cour de l'Empire romain à l'âge baroque (2018)

P. Arnaud, S. Ladstätter, F. Pirson, T. Schmidt (éds), Häfen und Hafenstädte im östlichen Mittelmeerraum (2014) 
A. Avram, J. Fournier et M.-G. G. Parissaki (éds), Les communautés du Nord égéen au temps de l'hégémonie romaine (2018)

Orient hellénistique et hellénisé

P. Briant, J.D. Lyons, Alexander the Great and Hernàn Cortés (2015)

P. Briant, K. Nawotka, A. Wojciechowka (éds), Alexander the Great and the East. History, Art, Tradition (2016)

P. Briant, S. Balatti, Mountain peoples in the Ancient Near East (2017)

$627-638$

Fr. Holt, O. Bordeaux, Les Grecs en Inde (2018)

L. Graslin-Thomé, K. Berthelot, In search of the promised Land? (2018)

S. BuIN, H. Kahwagi-Janho, Les monuments romains de Tyr extra muros (2016)

L. Berkes, A. Arjava, J. Frösen, J. Kaimio, The Petra papyri V (2018)

651-656

A. SARTRE-FAuriat, S. Krag, Funerary Representations of Palmyrene Women (2018)

657-660

$661-663$

A. Sartre-Fauriat, N.J. Andrade, Zenobia. Shooting Star of Palmyra (2018)

$665-667$

M.-O. Rousset, D. Dixneuf (éd.), LCRW 5 (2017)

$669-672$

\section{Orient}

Fr. JoAnnès, R. Pirngruber, The Economy of Late Achaemenid and Seleucid Babylonia (2017)

A. Nunn, B.Lafont, A.Tenu, Fr.Joannès, Ph.Clancier, La Mésopotamie. De Gilgamesh à Artaban (2017)

S. Gondet, L. Khatchadourian, Imperial Matter. Ancient Persia and the Archaeology of Empires, Oakland (2016)

G.L. Bonora, J. Lhuillier, N. Boroffka (éds), A Millennium of History. The Iron Age in southern Central Asia (2018)

V. Messina, V. Sarkhosh Curtis et al. (éds), The Parthian and Early Sasanian Empires (2016)

\section{Péninsule arabique}

J. Rohmer, A. Avanzini et M. Degli Esposti (éds), Husn Salut and the Iron Age of South East Arabia (2018)

\section{Égypte}

C. RÖMER, B. Van Beek, The Archive of the Architectones Kleon and Theodoros (P. Petrie Kleon) (2017)

G. TALLET, J.C.R. Gill, Dakhleh Oasis and the Western Desert (2016)

B. Van Oppen, E. Galbois, Images du pouvoir et pouvoir de l'image (2018)

J. Gascou, D. Frankfurter, Christianizing Egypt (2018)

G. RuFFInI, R.E. McConnell, Getting Rich in Late Antique Egypt (2017)

A. Boud'HORs, D. L. Brooks Hedstrom, Monastic Landscape of Egypt (2017)

E. Garel, J. Cromwell, Recording Village Life. A Coptic Scribe in Early Islamic Egypt (2017)

\section{Asie Centrale et Extrême-Orient}

V. LefÈvre, H.P. Ray (éd.), Buddhism and Gandhara (2018) 



\section{UN PROFESSEUR DE LETTRES CHRÉTIEN AU PAYS DE MOAB}

Des centaines d'inscriptions funéraires antiques de la Jordanie sont gravées sur ce qu'on a pris l'habitude d'appeler à la suite de Louis Robert des «pierres errantes», c'est-à-dire déplacées de leur lieu d'origine et de trouvaille ${ }^{1}$. Heureusement, quelques grands types sont aisément reconnaissables. Leur identification, garantie par la découverte d'autres inscriptions in situ, permet souvent de formuler des hypothèses assez solides quant à leur provenance. C'est le cas en particulier des bustes funéraires d'Abila de la Décapole, des stèles de basalte d'Umm al-Jimāl et du sud du Ḥawrān, des stèles de Samrā', de celles de Rihāā, de Madaba ou encore de Zoora. La série des stèles du plateau d'al-Karak, l'ancien pays de Moab (Fig. 1), se distingue clairement des autres du point de vue formel autant qu'épigraphique. Il revient à Reginetta Canova de l'avoir montré dans un ouvrage paru en 1954, Iscrizioni e monumenti protocristiani del paese di Moab. «C'est un travail sympathique », comme l'écrivaient Jeanne et Louis Robert dans leur recension du Bulletin épigraphique ${ }^{2}$. L'auteur, de son nom de jeune fille Regina Dal Zio (1906-1977), était l'épouse de Francesco Canova, médecin italien actif en Palestine et en Transjordanie entre 1935 et $1947^{3}$. À l'époque où son mari dirigeait l'hôpital italien d'al-Karak, elle avait mis à profit sa formation littéraire et philologique de l'Université de Padoue en effectuant des travaux de terrain en amateur entre 1936 et 1939. Son corpus réunit plus de 400 stèles funéraires, pour la plupart inédites, tout en faisant le bilan des recherches de ses prédécesseurs du $\mathrm{XIX}^{\mathrm{e}}$ siècle. Depuis sa publication, il continue de fournir une base indispensable pour l'étude des épitaphes chrétiennes de la région. Ce recueil permet de mettre en perspective les découvertes plus ou moins fortuites, qui se multiplient à l'heure actuelle et qui aggravent la dispersion de nombreuses pierres errantes dans les

1. Voir notamment RoBert 1966 (= RoBert 1990, p.637-671).

2. Bull.ép. 1956, 337.

3. BAGATTI 1977-1978.

Topoi 22 (2018)

p. $331-340$ 


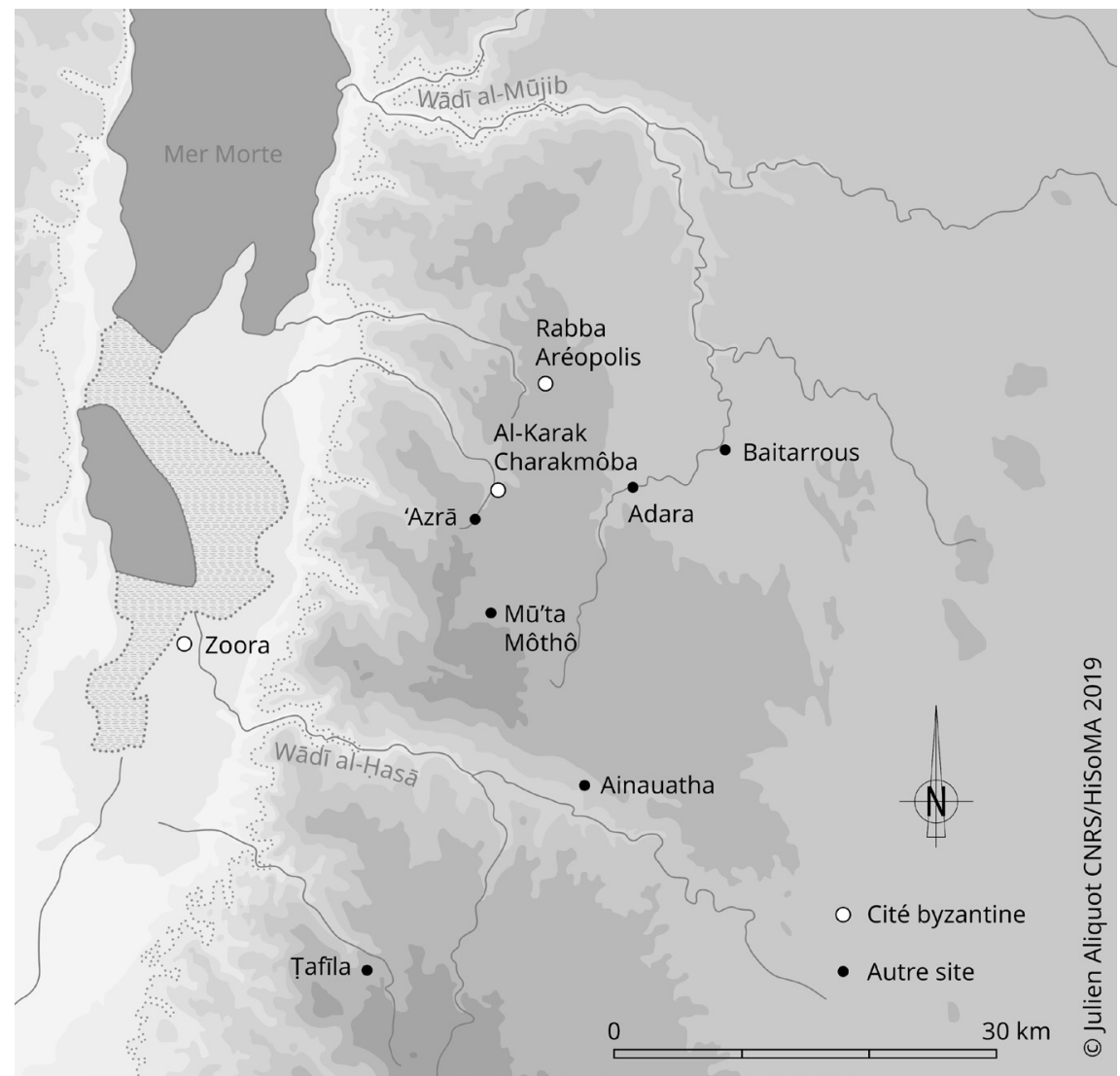

Fig. 1-Le pays de Moab (C) Julien Aliquot 2019).

musées et dans les collections privées ${ }^{4}$, tout en confirmant en partie les conclusions de Reginetta Canova.

On peut aujourd'hui évaluer le nombre des monuments funéraires du pays de Moab à environ 500. Ces stèles sont reconnaissables au premier coup d'œil. Taillées dans le calcaire dur local du plateau d'al-Karak, elles sont inscrites en grec et fréquemment ornées de symboles chrétiens. Leurs caractéristiques formelles, le formulaire de leurs inscriptions et l'onomastique des épitaphes permettent de les attribuer à la période comprise entre le milieu du IV siècle après J.-C. et le VII siècle après J.-C. Les dates que portent nombre d'entre elles doivent être converties dans le calendrier julien en utilisant l'ère provinciale de l'Arabie romaine, dont l'époque fut fixée au 22 mars 106 après l'annexion du royaume nabatéen et qui

4. Aliquot, Shdaifat et Weber 2014; Gatier, Bader, Aliquot, Sartre et Yon 2017, p. $347, \mathrm{n}^{\circ} 9$. 
fut universellement utilisée dans le pays de Moab comme partout ailleurs dans la province, y compris après l'intégration de ce secteur dans la province de Palestine Troisième, peut-être dès la première moitié du v siècle. La plus ancienne épitaphe datée remonte aux années 375/376 après J.-C.; les plus récentes ont été gravées sous le califat omeyyade (661-750). La plupart des documents datés sont du $\mathrm{VI}^{\mathrm{e}}$ siècle après J.-C. ${ }^{5}$.

Environ $5 \%$ du corpus de Moab est constitué d'épigrammes funéraires. Au moins 22 ou 23 inscriptions de ce type, en majorité d'al-Karak, l'antique cité de Charakmôba, ont été publiées à ce jour, dont 17 ou 18 par Reginetta Canova ${ }^{6}$. Il s'agit en général de tire-larmes sans originalité. La plupart se limitent à mettre en vers les épitaphes, pour exprimer l'idée que la tombe est celle d'Untel, mort à tel âge. On relève à l'occasion des emprunts à la poésie épique ou au répertoire de l'Anthologie grecque, mais souvent l'inclusion des noms de personnes locaux dans des schémas connus vient perturber la métrique. Dans l'ensemble, la production épigrammatique régionale témoigne de l'hellénisation tardive et imparfaite des notables des deux cités (Aréopolis/Rabathmôba, Charakmôba) et des villages antiques du plateau (Adara, Ainauatha, Baitarrous, Môthô, etc.).

L'objet de la présente contribution est l'étude d'une épigramme inédite, que les travaux récents de l'équipe des Inscriptions de la Jordanie ont permis de repérer à 'Ammān. Si la provenance exacte de ce document est inconnue, ses caractéristiques la rattachent de manière évidente au pays de Moab. Comme on le verra, notre inscription est plus sophistiquée que les autres épigrammes funéraires de la région. Elle sort aussi de l'ordinaire en révélant l'identité d'un poète méconnu en même temps qu'une dynastie de grammairiens locaux.

La stèle qui porte notre épigramme est conservée dans le centre-ville de 'Ammān, où nous l'avons vue en 2015 et en 2017 (Fig.2). Elle fait partie de l'ancienne collection de Gassān Rīhānī (marchand jordanien décédé en 2001), acquise par le propriétaire actuel du restaurant Zorba, où elle est exposée. Il s'agit d'une stèle funéraire en calcaire jaune, de forme parallélépipédique et au sommet

5. Pour la chronologie des stèles funéraires du pays de Moab, l'ouvrage de CANova 1954 doit être complété par la synthèse de MeIMARIs 1992 sur les inscriptions datées de l'Arabie et de la Palestine romaines et byzantines et par l'article de GATIER 2012 sur les documents épigraphiques d'époque islamique au Proche-Orient. Sur les remaniements territoriaux de l'Arabie et de la Palestine, voir en dernier lieu Di SEGNI 2018.

6. Canova 1954, $\mathrm{n}^{\text {os } 3}, 47,59,76,108,144,169,173,181,183,194$ (?), 210, 223, 275-277, 285, 302; Mouterde 1957, p.268 (SEG 19, 895; Bull. ép. 1959, 480); Aliquot, ShDaifat et Weber 2014, p. 150-151, nº 2 , et p.152-153, $\mathrm{n}^{\circ} 5$. Voir aussi Gatier 1992 ; Merkelbach et Stauber 2002, p. 436-444,454. On ajoutera à cette liste l'épitaphe versifiée de Kallinikos, reprise dans $S E G 58,1777$, conservée au musée archéologique de la Citadelle de 'Ammān et faussement attribuée à Philadelphie de la Décapole. Il faut peut-être prendre aussi en considération l'épigramme honorifique pour le général Dôrothéos, de provenance incertaine, dont FeISSEL 1984, p.545-546, 556-557, donne une nouvelle étude. 
arrondi, haute de $80 \mathrm{~cm}$, large de $49 \mathrm{~cm}$ et épaisse de $20 \mathrm{~cm}$. La face arrière, bombée, du monument est sommairement dégrossie. Sa face avant, inscrite, est mieux ravalée, sauf au pied de la stèle, dans la partie destinée à être enterrée. Elle présente un décor incisé qui, imitant l'architecture d'une niche, se compose de deux colonnettes stylisées supportant un arc cintré orné de zigzags. L'inscription est soigneusement gravée sur cette même face en lettres hautes de 3 à $3,5 \mathrm{~cm}$. Tout comme le décor, elle était rubriquée, d'après les traces de peinture qui subsistent çà et là. La première partie du texte occupe un bandeau situé sous l'arc, au-dessus de deux colombes adossées de part et d'autre d'une croix pattée. La seconde partie, versifiée, est gravée à la suite d'une croix, dans le champ rectangulaire de $61 \mathrm{~cm}$ de haut sur $41 \mathrm{~cm}$ de large délimité par un trait horizontal incisé sous l'arc, par les deux colonnettes et par le pied de la stèle. Le texte présente une abréviation signalée par la barre oblique qui traverse le kappa à la fin de la ligne 1, ainsi que de nombreuses ligatures et un signe de ponctuation à la fin de la ligne 8 . Les dernières lettres de la ligne 3 sont ajoutées en petits caractères de $2 \mathrm{~cm}$ de haut dans l'interligne suivant. À la fin de la ligne 4, le sigma est gravé dans la marge à droite.

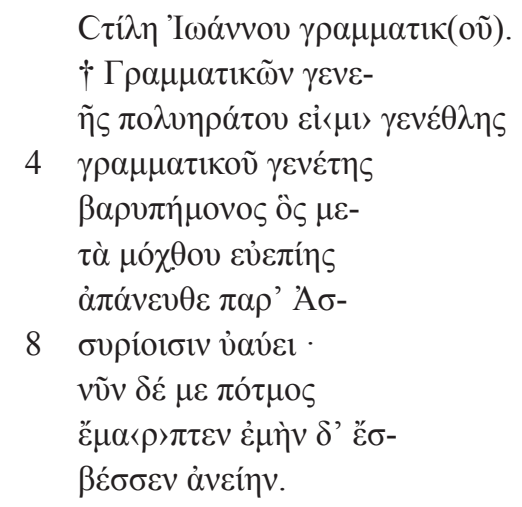

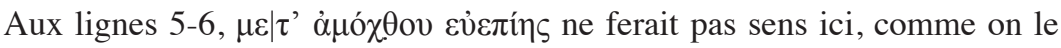
verra plus loin. On notera au passage que la barre médiane du théta de $\mu$ ó $\chi$ ovo n'est pas gravée, mais qu'elle a pu être peinte. Par ailleurs, deux autres erreurs graphiques et deux confusions phonétiques doivent probablement être attribuées au lapicide plus qu'à l'auteur du poème. Au début de la ligne 1 , la pierre porte $\sigma \tau i ́ \lambda \eta$ au lieu de $\sigma \tau \hat{\jmath} \lambda \eta$. À la ligne 3, comme me le suggère Denis Feissel, pour que le premier vers soit correct, il faut supposer l'omission du mu et du iota à la

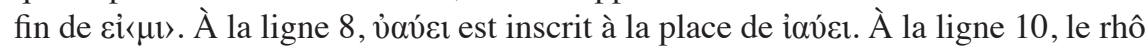
de $\varepsilon \tilde{\mu} \alpha<\rho>\pi \tau \varepsilon v$ est omis.

Voici l'épigramme présentée en vers, à la suite de l'épitaphe en prose de la première ligne:

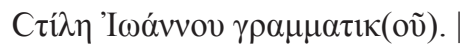

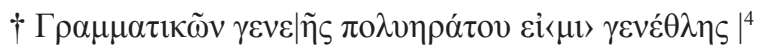

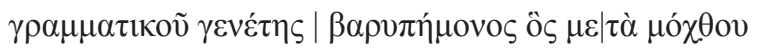




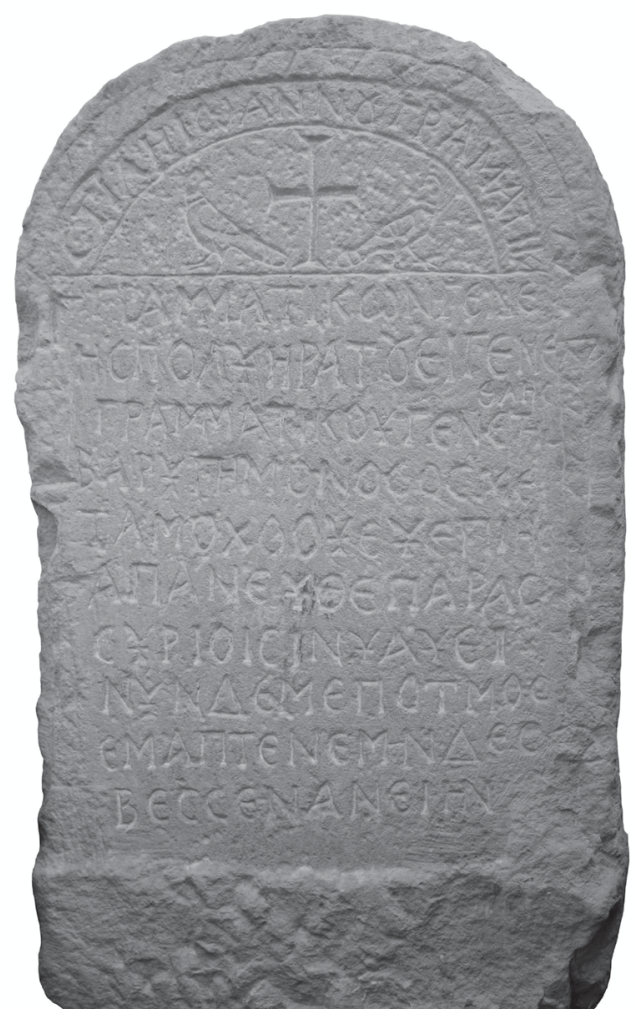

Fig. 2 - La stèle funéraire de Jean le Grammairien (C) Julien Aliquot 2015).

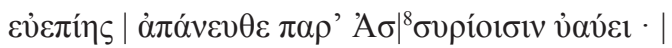

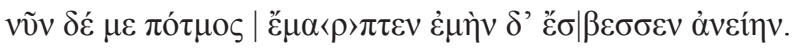

Traduction: «Stèle de Jean le Grammairien. D’une famille de professeurs de lettres à la très aimable descendance, je suis le père d'un malheureux grammairien qui, victime des affres du beau langage, repose au loin chez les Assyriens; désormais la mort s'est emparée de moi et a apaisé mon chagrin.»

L'épigramme se compose de quatre hexamètres de bonne facture. C'est une pièce originale, même si certains passages rappellent des formules connues ${ }^{7}$. Sur

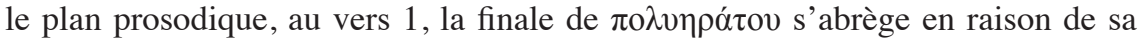

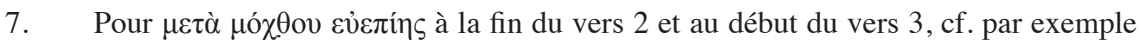
AP 9, 471, 1 ( $\mu \varepsilon \tau \grave{\alpha} \mu$ ó́ $\chi$ ov), ainsi que Nonnos de Panopolis, Dionysiaques 16, 134

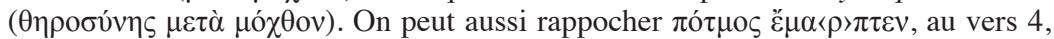

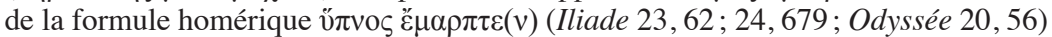

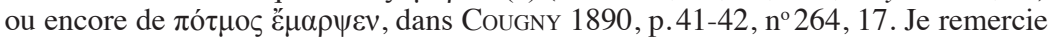
Gianfranco Agosti de m'avoir fait part de ses remarques sur ces deux passages. 
position en hiatus et sur le second temps faible d'un dactyle. À la fin du vers 4, on note encore la gémination du sigma dans le verbe $\sigma \beta \varepsilon ́ v v v \mu$ à l'aoriste ("̌ $\sigma \beta \varepsilon \sigma \sigma \varepsilon v$ pour $\check{\varepsilon} \sigma \beta \varepsilon \sigma \varepsilon v)$. Dans l'ensemble, ces procédés sont à porter au crédit d'un poète rompu aux techniques de la versification classique.

Le poème n'étant pas signé, son auteur n'est pas définitivement identifiable. Cependant, vu le pedigree et le métier du défunt ${ }^{8}$, il y a fort à parier que Jean a composé lui-même son épitaphe. Ce dernier porte un nom chrétien très banal, abondamment attesté dans la région d'al-Karak comme partout dans le monde byzantin. D'autres personnages appelés Jean le Grammairien sont connus dans l'Antiquité tardive. Le plus fameux est Jean Philopon ( $c$ a 490-574) ${ }^{9}$. L'expression

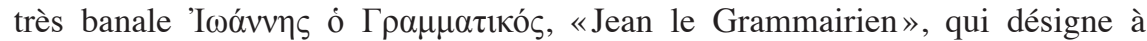
plusieurs reprises ce commentateur d'Aristote et théologien chrétien du $\mathrm{VI}^{\mathrm{e}}$ siècle, a d'ailleurs entraîné de nombreuses confusions avec des grammairiens homonymes. On trouvera leurs noms dans la partie prosopographique du livre de Robert A. Kaster, Guardians of Language, qui ne recense pas moins de sept ou huit grammairiens nommés Jean et souvent versés dans la poésie (dont le célèbre Jean de Gaza, cité de Palestine Troisième) ${ }^{10}$. Aucun rapprochement ne s'impose entre eux et notre humble professeur du pays de Moab.

Une autre originalité de l'épigramme est de porter à notre connaissance l'existence d'une famille entière de grammairiens locaux ${ }^{11}$. Ces derniers complètent la liste des grammairiens de l'Arabie et de la Palestine Troisième, liste très courte et pleine d'incertitudes, sans surprise, compte tenu de la condition sociale parfois médiocre des professeurs de lettres ${ }^{12}$, longtemps inférieure à celle des sophistes et des rhéteurs qui trônaient au sommet de la hiérarchie des études littéraires et qui pouvaient occuper de prestigieuses chaires publiques dans les cités. Tous n'ont probablement pas connu le même succès que Jean de Gaza (fl. 500-530), poète renommé et grammairien appointé par sa patrie ${ }^{13}$.

8. L'objet principal des leçons du grammairien est précisément l'étude approfondie des poètes et des auteurs classiques, inculquée à des élèves qui savent déjà lire et écrire. Voir MARRou 1965, p.243-244; KASTER 1988, p.11-14; WATTS 2012, p. 469.

9. Giardiana, Gannagé et al. 2012.

10. KASTER 1988, p. 298-301.

11. Sur les grammairiens dans les sources épigraphiques, outre l'ouvrage de KASTER 1988, voir la compilation d'Agusta-Boularot 1994, qui se concentre sur les quatre premiers siècles de l'ère chrétienne.

12. Voir à ce sujet tout la partie du livre de KASTER 1988 symptomatiquement intitulée «Mediocrity»,p.97-134.

13. LaURitzen 2015-2016, sur Jean de Gaza et sur la réévaluation du statut du grammairien employé de la cité aux $\mathrm{v}^{\mathrm{e}}-\mathrm{VI}^{\mathrm{e}}$ siècles. 
En 358 après J.-C., un certain Tibérinus contacta Libanios alors que son fils Archélaos faisait l'objet de poursuites en Arabie, sa province d'origine. Libanios écrivit alors au gouverneur Maximus que Tibérinus était «un homme excellent dans l'ensemble, notamment parce qu'il introduisait les poètes dans les esprits des

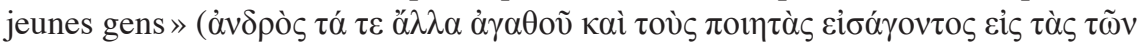

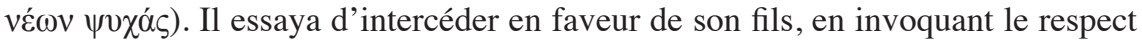
de Maximus pour la culture, pour les cités d'origine et d'adoption de Tibérinus et pour Libanios lui-même ${ }^{14}$. Par ailleurs, Joseph Geiger a proposé d'identifier, sans certitude, le sophiste Zénobios d'Élousa (Néguev), rhéteur officiel d'Antioche et maître de Libanios mort en 354, avec le grammairien homonyme à qui le poème $A P 9,711$ est attribué ${ }^{15}$. D'après la correspondance de Procope de Gaza, un certain Jérôme (Hiéronymos), lui aussi originaire d'Élousa, a pu être un grammairien ou plutôt un rhétoricien actif en Égypte, en particulier à Hermopolis ${ }^{16}$. Plus sûrement, un autre grammairien originaire d'Arabie, certainement chrétien, est Alexandros, dont l'épigramme funéraire a été retrouvée à Merdocha (Murduk) au nord-ouest de l'Auranitide ${ }^{17}$. On peut enfin citer, de manière plus anecdotique pour notre propos, les frères Graptoi, Théodôros (775-845) et Théophanès (778-844), nés à Karak dans le pays de Moab et devenus moines, qui furent tous deux initiés à la littérature classique (grammaire, philosophie, poésie) dans une école proche de l'église de la Résurrection à Jérusalem, avant que l'anarchie consécutive à la mort du calife Hārūn al-Rašīd (786-809) les pousse à s'exiler à Constantinople; leur éducation leur aurait valu le titre de $\gamma \rho \alpha \mu \mu \alpha \tau$ เкоí ${ }^{18}$.

Pour en revenir à notre inscription, ce document constitue à la fois l'épitaphe de Jean le Grammairien et un hommage post-mortem rendu à son fils, lui aussi grammairien et mort loin de sa patrie. L'évocation du fils de Jean rappelle la mobilité qui caractérise les professeurs de lettres du monde romain, du Haut-Empire jusqu'à la fin de l'Antiquité. Robert A. Kaster estime qu'un quart des 140 grammairiens dont le lieu d'exercice est connu dans l'Antiquité tardive ont émigré au cours de leur carrière ${ }^{19}$. Les plus grandes métropoles attiraient naturellement les maîtres originaires de cités moins populeuses et prestigieuses ou encore de régions retirées comme l'était le pays de Moab. Dans ce mouvement, des cités telles qu'Athènes

14. Libanios, Ep. 337; PLRE 1, p.913, s.v. Tiberinus; KASTER 1988, p. 367-368, nº 155 , s.v. Tiberinus.

15. GeIGer 2014, p.41, s.v. Zenobius 1 .

16. KASTER 1988, p.413-414, no 231, s.v. Hieronymus; GeIGER 2014, p.24, s.v. Hieronymus 1.

17. Merkelbach et Stauber 2002, p. 399, n²2/31/98; IGLS 16/1, 12.

18. Efthymiadis 1995, p.141-144.

19. KASTER 1988, p.126-128. 
et Antioche servaient de tremplins pour parvenir jusqu'aux centres de la culture impériale, Nicomédie sous Dioclétien, Rome et Constantinople au IV siècle et après. La correspondance de Libanios, on l'a vu, atteste que des grammairiens originaires de l'Égypte, de la Phénicie et de la Palestine, mais aussi de l'Arabie, étaient présents à Antioche au milieu du IV siècle.

Les deuxième et troisième vers de notre épigramme indiquent que le fils de Jean le Grammairien avait lui aussi pris le parti d'émigrer pour faire carrière, «lui qui, victime des affres du beau langage, repose au loin chez les Assyriens » (ò $\varsigma$

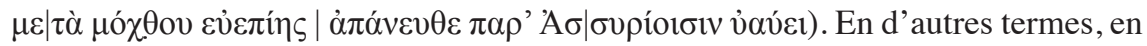
prenant le chemin de l'exil pour se consacrer à l'enseignement des belles-lettres ${ }^{20}$, il avait enduré les peines et les fatigues des grammairiens ${ }^{21}$. La mention du pays des Assyriens ne doit pas tromper. Plutôt qu'une allusion bien improbable à la Mésopotamie et aux confins des empires byzantin et perse, il faut y reconnaître la dénomination poétique et savante classique de la Syrie, bien attestée encore dans l'Antiquité tardive, par exemple dans les Dionysiaques de Nonnos de Panopolis ${ }^{22}$. On peut se demander si le fils de Jean n'avait pas enseigné la littérature grecque à Antioche, cité syrienne par excellence, où la demande en professeurs était susceptible d'attirer des maîtres venus d'Arabie et de Palestine Troisième. Cela restera sans doute une hypothèse.

En 1954, Reginetta Canova en arrivait à la conclusion suivante, à propos de la poésie épigrammatique du pays de Moab $^{23}$ : «D'un côté, celle-ci constitue un témoignage intéressant de la diffusion de la culture grecque et des expressions classiques jusqu'aux confins du désert, mais de l'autre elle est la preuve qu'une telle diffusion était uniquement superficielle: elle imitait, mais n'assimilait pas. La connaissance même des formes littéraires devait être limitée à un nombre très restreint de personnes et nous devons penser que des mots d'usage rare et

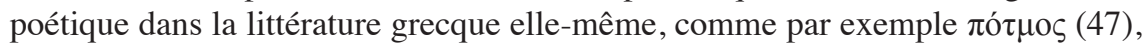

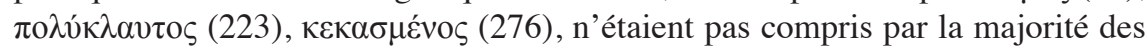
fidèles et étaient introduits dans les épitaphes par des personnes d'une certaine culture qui, sans pour autant avoir une instruction littéraire grecque complète, en gardaient un lointain souvenir scolaire ou, plutôt, qui tiraient de recueils et d'anthologies des expressions et des formules à des fins pratiques, car autrement il

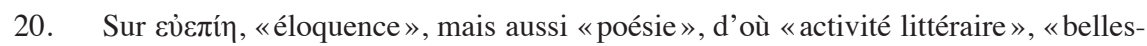
lettres», «humanités» au sens large, voir les parallèles réunis par MAGNELLI 2004, p. 272 n. 15.

21. Lieu commun développé par exemple chez Palladas, poète et grammairien alexandrin du $\mathrm{IV}^{\mathrm{e}}$ siècle. Voir KASTER 1988, p.327-329, n 113, s.v. Palladas (notice biographique); WiLKINSON 2012 (épigrammes du papyrus de Yale récemment attribuées au même auteur).

22. Chuvin 1991,p.191-192; cf. NöLDEKe 1871.

23. Canova 1954, p.LXXVIII (ma traduction). 
ne serait pas possible d'expliquer la coïncidence d'erreurs grossières et de termes inhabituels qui n'apparaissent que dans la poésie.» Depuis plus d'un demi-siècle, on a appris à éviter d'évaluer à l'aune des pièces les plus raffinées de la littérature classique les productions poétiques régionales de l'Antiquité tardive, telles que les inscriptions les font connaître dans les différentes parties du monde antique ${ }^{24}$. L'attachante étude des épigrammes du pays de Moab ne donne d'ailleurs qu'en partie raison à Reginetta Canova. L'épitaphe de Jean le Grammairien conduit ainsi à nuancer son propos tout en nous plongeant au cœur du petit monde des maîtres d'école, des professeurs de littérature grecque et des poètes mineurs, qui pouvaient eux aussi faire preuve d'originalité et même d'un certain talent.

\section{Julien Aliquot CNRS, HiSoMA, Lyon}

\section{Bibliographie}

Agusta-Boularot S. 1994, «Les références épigraphiques aux grammatici et $\gamma \rho \alpha \mu \mu \alpha \tau$ roí de l'Empire romain ( $\mathrm{I}^{\mathrm{er}} \mathrm{s}$. av. J.-C. - IV siècle apr. J.-C.) », MEFRA 106, p.653-746.

Aliquot J., Y. Shdaifat et T.M. Weber 2014, «New Byzantine Inscribed Tombstones from the Land of Moab», ZPE 189, p. 149-158.

Bagatti B. 1977-1978, «In Memoriam Reginetta Canova, a Pioneer Archeologist in Jordan», ADAJ 22, p. 181-182.

CANova R. 1954, Iscrizioni e monumenti protocristiani del paese di Moab, Sussidi allo studio delle antichità cristiane 4, Rome, Cité du Vatican.

Chuvin P. 1991, Mythologie et géographie dionysiaques. Recherches sur l'œuvre de Nonnos de Panopolis, Clermont-Ferrand.

Cougny E. 1890, Epigrammatum Anthologia Palatina cum Planudeis et Appendice nova epigrammatum veterum ex libris et marmoribus 3, Paris.

Di SEgNI L. 2018, «Changing Borders in the Provinces of Palaestina and Arabia in the Fourth and Fifth Centuries », Liber Annuus 68, p.247-267.

Efthymiadis S. 1995, «Notes on the Correspondence of Theodore the Studite», REB 53, p.141-163.

Feissel D. 1984, «Notes d'épigraphie chrétienne (VII)», BCH 108, p.545-579.

Gatier P.-L. 1992, «Répartition des inscriptions grecques de Jordanie. L'exemple des inscriptions métriques aux époques romaine et byzantine», Studies in the History and Archaeology of Jordan 4, p. 291-294.

GATIER P.-L. 2012, «Inscriptions grecques, mosaïques et églises des débuts de l'époque islamique au Proche-Orient ( $\mathrm{VII}^{\mathrm{e}}-\mathrm{VIII}^{\mathrm{e}} \mathrm{s}$.)», in A.Borrut, M.Debié, A.Papaconstantinou, D. Pieri et J.-P. Sodini (éds), Le Proche-Orient de Justinien aux Abbassides, Bibliothèque de l'Antiquité tardive 19, Turnhout, p.7-28.

24. Voir supra, p. 305-329, les observations de Gianfranco Agosti sur les épigrammes de la Jordanie. 
Gatier P.-L., N. Bader, J.Aliquot, M. Sartre et J.-B. Yon 2017, «Greek Inscriptions in the Jordan Museum», ADAJ 58, p.341-350.

GeIGer J. 2014, Hellenism in the East. Studies on Greek intellectuals in Palestine, Historia Einzelschriften 229, Stuttgart.

Giardiana G.R., E. Gannagé et al. 2012, «Philopon (Jean -)», in R.Goulet (éd.), Dictionnaire des philosophes antiques 5a. De Paccius à Plotin, Paris, p.455-563.

KASTER R.A. 1988, Guardians of Language. The Grammarian and Society in Late Antiquity, Berkeley, Los Angeles, Londres.

Lauritzen D. 2015-2016, «Sur l'identité de Jean de Gaza. I. - Grammatikos et notable», Revue des études tardo-antiques 5, p.177-210.

Magnelli E. 2004, «App. Anth. II 400 C.: Oppiano come Erinna?», Prometheus 30, p.269274.

Marrou H.-I. 1965, Histoire de l'éducation dans l'Antiquité, 6e éd., Paris.

Meimaris Y.E. 1992, Chronological Systems in Roman-Byzantine Palestine and Arabia.

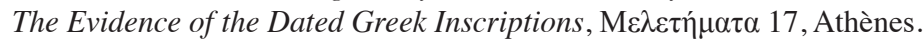

Merkelbach R. et J. Stauber 2002, Steinepigramme aus dem griechischen Osten 4. Die Südküste Kleinasiens, Syrien und Palaestina, Munich, Leipzig.

MOUTERDE R. 1957, «Dott. Reginetta Canova, Iscrizioni e monumenti protocristiani del paese di Moab (Sussidi... per la cura del Pontificio Istituto di Archeologia Cristiana, IV). Gr. In- $8^{\circ}$, CXXIX-438 pp. Cita del Vaticano, 1954», Mélanges de l'Université Saint-Joseph 34, 1957, p.266-268.

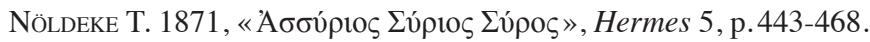

ROBERT L. 1966, «Pierres errantes, muséographie et onomastique», Berytus 16, p.5-39.

ROBERT L. 1990, Opera minora selecta 7, Amsterdam.

Watts E. 2012, «Education: Speaking, Thinking, and Socializing», in S.F. Johnson (éd.), The Oxford Handbook of Late Antiquity, Oxford, p.467-486.

Wilkinson K.W. 2012, New Epigrams of Palladas. A Fragmentary Papyrus Codex (P.CtYBR inv. 4000), American Studies in Papyrology 52, Durham, NC. 